\title{
Privacy Protection in Cloud computing by using Cryptographic Technique.
}

\author{
Rajapraveen. k. $\mathrm{n}^{1}$, Dr. N. K. Prasanna kumari ${ }^{2}$ \\ ${ }^{I}$ (M.tech/Department of computer science \& engineering, sset/Sam Higginbottom Institute of Agriculture, \\ Technology \& Sciences-deemed University, India) ${ }^{2}$ (NIMH, Nagpur)
}

\begin{abstract}
Cloud computing technology is very useful in present day to day life, this cloud computing techniques uses the internet and central remote servers to the maintain data as well as applications. Cloud computing permits consumers and businesses to use such applications without getting any installation and can access their data (or) files from any other computer using internet service. Cloud computing Technology provides vast and better efficient computing by centralizing data storage, processing. The work of cloud computing is to store, create, and manipulate data through Web based services, due to the flexi services by cloud computing it is growing in popularity. But when coming to privacy factor, the question arises these cloud computing services can able to protect the privacy of their consumers, What are the necessary measurements and what precautions we can do for the improvement of privacy .to enhance the privacy in cloud computing cryptographic techniques are more useful.
\end{abstract}

Keywords: cryptographic protection in cloud computing, privacy in cloud computing, privacy protection with cryptographic technique in cloud computing

\section{Introduction}

Cloud computing services, accessed through Internet that allow consumers to create, edit, and store documents such as private photos and videos, calendars and address books, e-books and journals, and budgets and financial spreadsheets in online. most of the existing Companies providing a wide range of cloud computing services that ranges from "free" basic applications for the general public to sophisticated and wellsupported services designed for corporations sector as well as for government sector, Many popular offline applications, including Microsoft Office and Adobe Photoshop. Cloud computing is editions of familiar interfaces, tools allow consumers to "drag and drop" files to or from online. Consumers can easily access and share's the accessed data from any Internet-enabled device. From consumer perspective, information cannot be carried out or information can't get into the cloud easily, the privacy protections for that personal information may not transition as easily.

A cloud can be of two types 1) private or 2) public. A public cloud can send services to anyone on the Internet. (EX: - Amazon Web Services is the largest public cloud provider.) A private cloud is a proprietary network (or) data center that provides the services to a limited number of people. When a service provider uses public cloud resources to create their private cloud, the result is called a virtual private cloud. Private or public, the goal of cloud computing is to provide easy, scalable access to computing resources and IT services.

\section{Privacy On Cloud Computing}

Cloud computing services can hold a consumer's, business records, photographs, calendars ,address books, e-books, medical records, and many other sensitive documents that the consumer regards as Private. Information that contains in such documents can implicate every part of a consumer's life his (or) her family and friends, politics and religion, interests and activities - that requires significant safeguards to protect his (or) her privacy and independence of action.

\section{Necessary Steps To Solve Privacy Problem}

On taking necessary steps towards cloud computing activities like any Internet activity that generating additional information that a provider might collect, such as

$>$ The identity of each consumer who accesses content that stored in online and the time, place they do so.

Ex: - when a consumer is accessing Google Docs, Google records information such as Account activity (ex: storage usage, number of log-ins, actions taken), data displayed or clicked on (ex: UI elements, links), and other log information (ex: browser type, IP address, date and time of access, cookie ID, referrer URL). On Collecting this information raises questions about privacy even when done independently; when linked to other cloud computing activity, it threatens to reveal far more about a consumer than he (or) she might imagine. 
Ex: - IP addresses and login times could be used to determine when and where a user was and who else has used that concerned computer if he (or) she logs into a cloud computing service away from home.

\section{Privacy Policies}

Internet consumers are looking for high security control over their own information. Consumers were very much concerned about all the possibility that their personal information could be shared with the help of law enforcement (or) any other third parties.

Technology should play an important role in helping to safeguard the privacy. Right Now, consumers are very concerned about their information being used in ways that they did not intend. This Concern is not good for the public this is the time for technology to ensure trusts on cloud computing because more people will use this technology if they trust that their personal information will remain Private and their information will be safe if they use a cloud computing service.

\section{Existing Techniques:-}

Designing services with technical measures to protect consumer's tools that allow consumers to manage and protect their own information, encryption and anonymity protocols are used to protect information by default, and access controls and data security measures to prevent breaches and inappropriate disclosures cloud computing providers can establish a platform where consumers are in a position To control their own information and can feel more confident in storing private content.

\section{My Contribution To Protect Privacy In Cloud Computing Services}

My contribution on providing the privacy by using cryptographic technique of encryption, encryption is of two types

a) Symmetric encryption: - private key encryption

b) Asymmetric encryption: - public key encryption

$>$ Symmetric encryption technique is more useful in achieving the protection of Privacy in cloud computing.

$>$ Symmetric encryption is also called as private key encryption (or) single key encryption.

$>$ It is oldest type of encryption used in(1970)

$>$ Same key must use to encrypt (or) decrypt the data

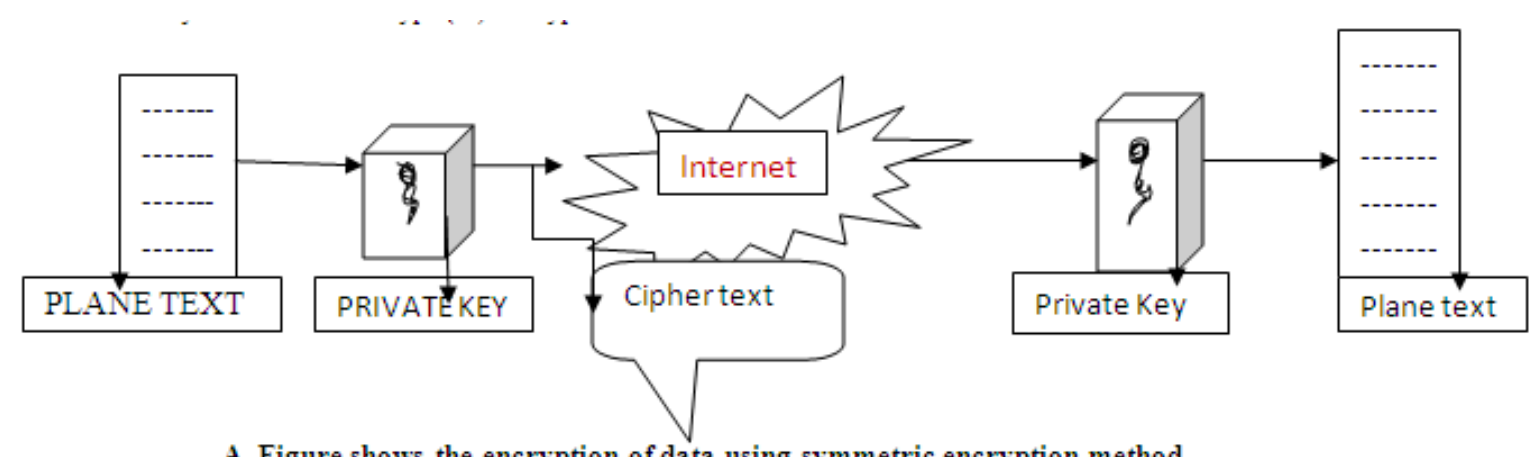

A. Figure shows the encryption of data using symmetric encryption method

Plane text - this is original data that is fed into the encryption algorithm as input Encryption algorithm Encryption algorithm performs various substitutions and transformations on the plane text.Secret key - Secret key is also input to the algorithm. The exact substitution's and transformations performed by the algorithm depends on the key.Cipher text - this is scrambled data produced as output; it depends on the plane text and secret key for a given data.

Decryption algorithm - this is essentially the encryption algorithm run in reverse, it takes cipher text and the same secret key produces the original plane text.

By applying this symmetric encryption technique to cloud computing, the personal information can be encrypted by using symmetric encryption technique (or) private key (or) single key technique, this single key (or) private key cannot shared by others for safety measurement . This private key is used for encrypting and decrypting of data. We can achieve protection of privacy in cloud computing; the personals of his (or) her should be in controlled way, when unauthorized party tried to access the data (or) files etc... In cloud computing, third parties will ask for the private key, for accessing. 


\section{Acknowledgement}

I sincerely convey my thanks to my beloved Vice - Chancellor \& our founder first Bishop YESHU DARBAR, Rev. Fr. Prof.(Dr.) Rajendra B. Lal for his guidance, support and help, and I also convey my thanks to my mother and grandmother Mrs.Swarnalatha.N \& M. Amulya Prema for their parental guidance.

\section{Conclusion}

Cloud computing provides various advantages and offers many facilities for users, but due to lack of Inadequate privacy policies and lack of technological tools privacy factor in cloud computing become low, but with the help of our Privacy protection in Cloud computing by using cryptographic technique, privacy protection can be achieved in cloud computing services.

\section{References}

[1] Sun Microsystems, Introduction to Cloud Computing Architecture, 2009

[2] CHAPPELL, D.2009. Introducing the Azure Services Platform. David Chappell \& Associates.

[3] cloud computing http://www.wikinvest.com/concept/Cloud_Computing

[4] Dave Dittrich, Network monitoring/Intrusion Detection Systems (IDS), University of Washington

[5] M. Bellare, A. Desai, E. Jokipii, and P. Rogaway. A Concrete Security Treatment of Symmetric Encryption: Analysis of the DES Modes of Operation. Proceedings of the $38^{\text {th }}$ Symposium on Foundations of Computer Science, IEEE, 1997. 